\title{
Artefacts in cone beam CT
}

\author{
Prashant P. Jaju, Mayuri Jain, Ajita Singh, Akanksha Gupta
}

Rishi Raj College of Dental Sciences and Research Centre, Bhopal, India

Email: docprashant_jaju@yahoo.com

Received 22 May 2013; revised 23 June 2013; accepted 17 July 2013

Copyright (C) 2013 Prashant P. Jaju et al. This is an open access article distributed under the Creative Commons Attribution License, which permits unrestricted use, distribution, and reproduction in any medium, provided the original work is properly cited.

\begin{abstract}
Cone beam computed tomography (CBCT) is the modern third dimension applied in the field of oral maxillofacial region. With lower radiation dose compared to conventional CT, its applications in dentistry has increased tremendously. Artefacts can seriously degrade the quality of computed tomographic (CBCT) images, sometimes to the point of making them diagnostically unusable. To optimize image quality, it is necessary to understand why artifacts occur and how they can be prevented or suppressed. CT artifacts originate from a range of sources; physical based, scanner based and patient based. This article highlights the causes of artefacts on CBCT images and methods to avoid them.
\end{abstract}

Keywords: CBCT; Artefacts; Metal Artefacts

\section{INTRODUCTION}

The introduction of Cone Beam Computed Tomography (CBCT) technology in dentistry is rapidly changing the diagnostic landscape, allowing dentists to now diagnose in three dimensions. Present state-of-the-art cone beam computed tomography (CBCT) units produce excellent high resolution, three dimensional images of oral bony anatomy, making dental implant planning and surgical placement simple and reliable. Also the role of CBCT in oral \& maxillofacial surgery, orthodontics, airway assessment, temporomandibular joint disorders, endodontics and periodontics is widely described lately [1-6]. The radiation dose required for CBCT is lower than that of CT if we consider images made for the same purposes $[7,8]$.

In computed tomography (CT), the term artefact is applied to any systematic discrepancy between the CT numbers in the reconstructed image and the true attenuation coefficients of the object. CT images are inherently more prone to artefacts than conventional radiographs because the image is reconstructed from something on the order of a million independent detector measurements [9].
The presence of grey level non-uniformities in CBCT contributes to artifact formation in reconstructed CBCT images [10]. These artefacts contribute to image degradation and can lead to inaccurate or false diagnoses. Some of these artefacts are more pronounced in CBCT units than their CT counterparts because of the different processes in which the images are acquired (Figure 1).

Artefact relating to CBCT will be divided into three main categories, physics-based, patient-based and scanner-based. Physics-based artifacts result from the physiccal processes involved in the acquisition of CT data. Patient-based artifacts are caused by factors related to the patient's form or function. Scanner-based artifacts result from imperfections in scanner function.

\section{PHYSICAL BASED ARTEFACTS}

\subsection{Noise}

Noise is defined as an unwanted, randomly and/or nonrandomly distributed disturbance of a signal that tends to obscure the signal's information content from the observer. Noise affects images produced by cone beam CT units by reducing low contrast resolution, making it difficult to differentiate low density tissues thereby reducing the ability to segment effectively. The noise in traditional projection radiography is primarily from quantum mottle which is defined as a variation in image density due to statistical fluctuation of photon fluency in the radiation field. In well-designed X-ray systems, the quantum noise is governed by the number of X-ray photons absorbed in the detector, the higher the number of photons absorbed, the lower the quantum mottle. The number of X-ray photons emitted is directly related to the $\mathrm{mA}$ of the X-ray unit. Another source of noise in computed tomography is scattered radiation. Scattered radiation arises from interactions of the primary radiation beam with the atoms in the object being imaged and its magnitude is largely dependent on patient size, shape, and position in the scan field. It is a major source of image degradation in X-ray imaging techniques. When X-ray 


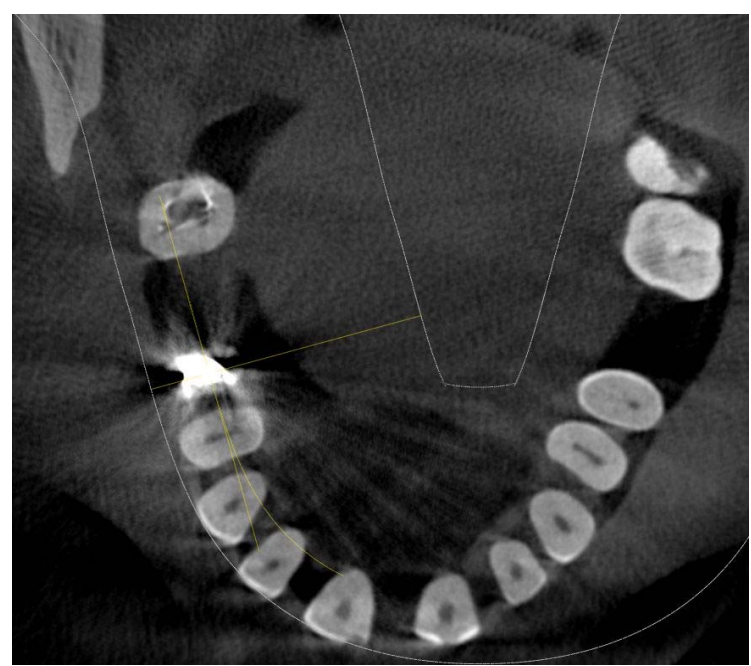

Figure 1. Artefact causing image degradation.

radiation passes through a patient, three types of interactions can occur, including coherent scattering, photoelectric absorption and Compton scattering. Compton scattering is the type most seen in diagnostic radiology. In Compton scattering, the interaction is a collision between a high energy X-ray photon and one of the outer shell electrons of an atom. This outer shell electron is bound with very little energy to the atom so when the X-ray photon collides with it, the electron is ejected from the atom. Because energy and momentum are both conserved in this collision, the energy and direction of the scattered X-ray photon depend on the energy transferred to the electron. If the initial X-ray energy is high, the relative amount of energy lost is small, and the scattering angle is small relative to the initial direction. If the initial $\mathrm{X}$-ray energy is small, the scatter angle is large and the ejected electron disperses in all directions. Quantum noise is fundamentally related to image quality and is a function of dose, tissue transmissivity, and voxel size. Noise is, in turn, a principal determinant of contrast resolution and, to a lesser extent, spatial resolution, which, along with artifacts, constitute the major observable determinants of overall image quality (Figure 2). CBCT imaging with flat panel detector (FPD) technology typically affords excellent spatial resolution with a relatively low patient dose. Contrast resolution suffers, however, due to increased X-ray scatter and the reduced temporal resolution and dynamic range of the FPDs [11]. Increased scatter not only amplifies patient dose but is a principal contributor to reduced contrast resolution and increased noise in CBCT images [11]. There is very little noise in conventional CT machines because of the high $\mathrm{mA}$ used and due to effective pre- and post patient collimation which reduces the scattered radiation to a negligible amount. However, in CBCT machines the noise is high due to the lower $\mathrm{mA}$ used and because of the high

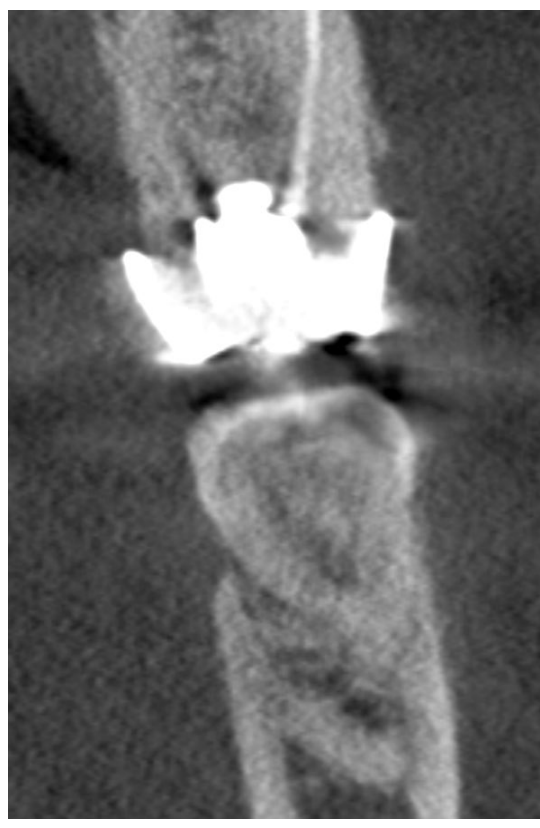

Figure 2. Noise reducing image contrast.

amount of scattered radiation since there is no post-patient collimation.

\subsection{Beam Hardening}

An X-ray beam is composed of individual photons with a range of energies. As the beam passes through an object, it becomes "harder," that is to say its mean energy increases, because the lower energy photons are absorbed more rapidly than the higher-energy photons. Two types of artifact can result from this effect: so-called cupping artifacts and the appearance of dark bands or streaks between dense objects in the image [9].

Beam hardening manifests as two different artifacts within the reconstructed image, a cupping artifact and the appearance of dark bands or streaks.

Cupping artifacts from beam hardening occur when $\mathrm{X}$-rays passing through the center of a large object become harder than those passing through the edges of the object due to the greater amount of material the beam has to penetrate. Because the beam becomes harder in the center of the object, the resultant profile of the linear attenuation coefficients appears as a “cup”. The cupping effect artifact is demonstrated when a uniform cylindrical object is imaged. As the effects of beam hardening and scatter are most prevalent in the centre of a cylindrical object, it is this area that is dominated by the cupping effect artifact [10].

The second type of artifact relating to beam hardening are dark streaks and bands between dense objects in an image. In dental imaging, this type of artifact can be seen between two implants located in the same jaw that are in close proximity to each other. This occurs because the 
portion of the beam that passes through both objects at certain tube positions becomes harder than when it passes through only one of the objects at other tube positions. McDavid et al. and Brooks and Di Chiro demonstrated that the cupping effect is caused by beam hardening by reconstructing a uniform object with ideal projections and observing the absence of the cupping effect $[12,13]$.

It is well known that an artifact referred to as the "truncation artifact" or "truncated-view artifact" is inherent to CBCT imaging. This artifact occurs because the size of the FOV used in CBCT is smaller than the size of the object being imaged. The largest error due to the truncated-view artifact will occur near the edge of the FOV [14]. Lehr imaged a $52 \mathrm{~cm}$ water disc phantom centred in a $48 \mathrm{~cm}$ FOV, which resulted in an increase in CT numbers at the edge of the FOV [14].

Bryant et al. described a similar observed effect, termed the "exomass effect", on the i-CAT (Imaging Sciences International, LLC, Hatfield, PA) CBCT unit [15].

They also observed an increase in the grey level values in the anterior to the posterior direction of the scan field, the posterior representing the position within the scan field, adjacent to the portion of the object located outside of the FOV. Katsumata et al. evaluated the effect of the truncation artifact on the Alphard Vega CBCT unit (Asahi Roentgen, Kyoto, Japan), reporting improved uniformity of the density values with the larger FOVs used [16].

Manufacturers minimize beam hardening by using filtration, antiscatter grids, calibration correction, and beam hardening correction software [9].

\subsection{Filtration}

The use of filtration to decrease beam hardening is supported by the findings of Brooks and Di Chiro, who demonstrated a reduction of beam hardening effects from $9.2 \%$ in $20 \mathrm{~cm}$ of water using a $4.5 \mathrm{~mm}$ aluminium prefilter to $1.5 \%$ using a $3.5 \mathrm{~cm}$ aluminium pre-filter and reported that using high atomic number ( $\mathrm{Z}$ ) materials such as copper, tin or Thoraeus filters could produce even better results [13]. In fact, normal aluminium filters are approximately $10 \%$ less efficient than filters of other materials such as copper, brass or iron [17]. Meganck et al. reduced the cupping effect caused by beam hardening on a cortical bone-equivalent phantom to an insignificant level (2\%) using a combination of $0.254 \mathrm{~mm}$ aluminium and $0.254 \mathrm{~mm}$ copper filter [10,18].

The bow tie or wedge filter is the prototypical compensating filter used in CBCT systems. It modulates the beam profile by increasing photon density at the center of the cone and decrementally reducing density at the periphery. In the radiation therapy CBCT literature, Graham et al. were able to demonstrate a 50\% reduction in scatter with the implementation of copper bow tie filters [19]. Image-quality improvement has been described with bow tie filters in a CBCT system integrated into the gantry of a conventional CT scanner as well [20].

Compensating filtration is not without criticism, however, because beam hardness hasbeen shown to negatively impact detector efficiency, as demonstrated by a decrease in the ratio of the output signal intensity-tonoise ratio (SNR) to the entrance exposure (SNR/entrance exposure) [21].

\subsection{Antiscatter Grids}

Antiscatter grids represent an alternative method of direct scatter reduction that has been used with FPDs in digital radiographic and fluoroscopic imaging for some time [22]. Rather than modulating the beam properties at the source, a grid of lead leaves is fitted over the detector to preferentially absorb off-axis radiation not contributing to primary photon fluence.

In CBCT systems, the lead leaves are arranged in a radial pattern centered on the focal spot of the FPD. Antiscatter grids have been evaluated in several experimental CBCT systems with mixed results [11,23,24]. A reduction in both cupping artifact and overall scatter has been observed, though there may be insufficient improvement in contrast and observed image quality to warrant use except in situations of high scatter [11]. Siewerdsen et al. evaluated antiscatter grids in a linear accelerator-coupled CBCT system and found that image quality improved only in situations of high scatter such as with a large FOV s covering a large anatomic site or in input quantum-limited situations such as with high dose or low spatial resolution [23]. To the extent that antiscatter grids improve soft-tissue contrast and artifacts, they also increase noise, which leads to a degradation in overall image quality. An escalation in dose or reduction in spatial resolution is needed to offset the increased noise with the implementation of grids. For a relatively small FOVs, such as that used in a targeted head and neck scan, antiscatter grids may improve image contrast and reduce cupping artifacts, but the increased noise requires that the dose be increased or spatial resolution be decreased to produce a high-quality image.

\subsection{Calibration}

Calibration correction: Manufacturers calibrate their scanners using phantoms in a range of sizes. This allows the detectors to be calibrated with compensation tailored for the beam hardening effects of different parts of the patient [9].

\subsection{Software Corrections}

Beam hardening correction software: An iterative correc- 
tion algorithm may be applied when images of bony regions are being reconstructed. This helps minimize blurring of the bone-soft tissue interface in brain scans and also reduces the appearance of dark bands in nonhomogeneous cross sections [9].

\subsection{Scatter Correction Algorithms}

Some sort of scatter subtraction or homogenization preprocessing algorithm is used in most clinical CBCT systems $[25,26]$. Several approaches have been studied, including Monte Carlo simulations, blocker-based or beamstop techniques, analytic calculations, and collimator shadow estimation [27]. Perhaps the most theoretically robust algorithm is that based on the Monte Carlo simulation, which predicts scatter on the basis of a voxel density model of the entire acquired tissue volume during preprocessing. The predicted scatter contribution at each detector element is then subtracted before reconstruction. Monte Carlo simulations still require significant computation time, however, which has fueled continued research in other algorithmic approaches.

\subsection{Partial Volume Artefacts}

The algorithms used in CT data reconstruction assume that the object is completely covered by the detector at all view angles, and that the attenuation is caused by the object only. When this situation does not occur, reconstructed CT images can contain a truncated-view artifact. In conventional CT units, this is not a problem as the entire object is always within the field of view of the unit, however it does affect CBCT units due to their limited FOV. This occurs because some of the cone beam data penetrating portions of the object other than the regionof-interest (ROI) are missing because of the insufficient size of the detector. When the entire volume is not covered by the detector, shading artifacts can be visualized. Another consequence of the partial volume artifact is that the true linear attenuation coefficients cannot be calculated because some of the X-ray paths penetrate other portions of the object as well as the region of interest and the data collected no longer represent this area exclusively but are corrupted by structures outside of the FOV. This issue has a greater affect in machines that have smaller FOVs as opposed to those that have larger FOVs. Currently, algorithms attempt to counter this issue by estimating the remaining linear attenuation coefficients for the areas that are not completely imaged. Although there is improvement in HU precision, this still has not enabled accurate calculation of Hounsfield units. Many methods are currently being developed and tested to alleviate this issue. Partial volume artifacts can best be avoided by using a thin acquisition section width [9].

\section{PATIENT-BASED ARTEFACTS}

\subsection{Metal Artefacts}

The presence of metal objects in the scan field can lead to severe streaking artifacts. They occur because the density of the metal is beyond the normal range that can be handled by the computer, resulting in incomplete attenuation profiles. Metallic objects such as dental restorations, surgical plates, dental implants and pins and radiographic markers can cause this type of the artifact. Since the metal in these materials highly attenuate the $\mathrm{X}$-ray beam, the attenuation values of objects behind the object are incorrectly high (Figure 3). Due to the reconstruction of the cone beam image, the metal causes the effect of bright and dark streaks in CT images which significantly degrade the image quality. In conventional CT images metallic artifacts traverse the object in the direction of the gantry and only at the level of the high attenuation object. In CBCT, the metallic streak artifacts occur in all directions from the high attenuation object because of the cone-shaped beam (Figure 4).

\subsection{Software Corrections for Metal Artifacts}

Streaking caused by overranging can be greatly reduced by means of special software corrections. Manufacturers use a variety of interpolation techniques to substitute the overrange values in attenuation profiles. MARS (Metal artifact reduction software) provided by Sirona, (Germany) is one such metal reduction software which improves the quality of image. The usefulness of metal artifact reduction software is sometimes limited because, although streaking distant from the metal implants is removed, there still remains a loss of detail around the

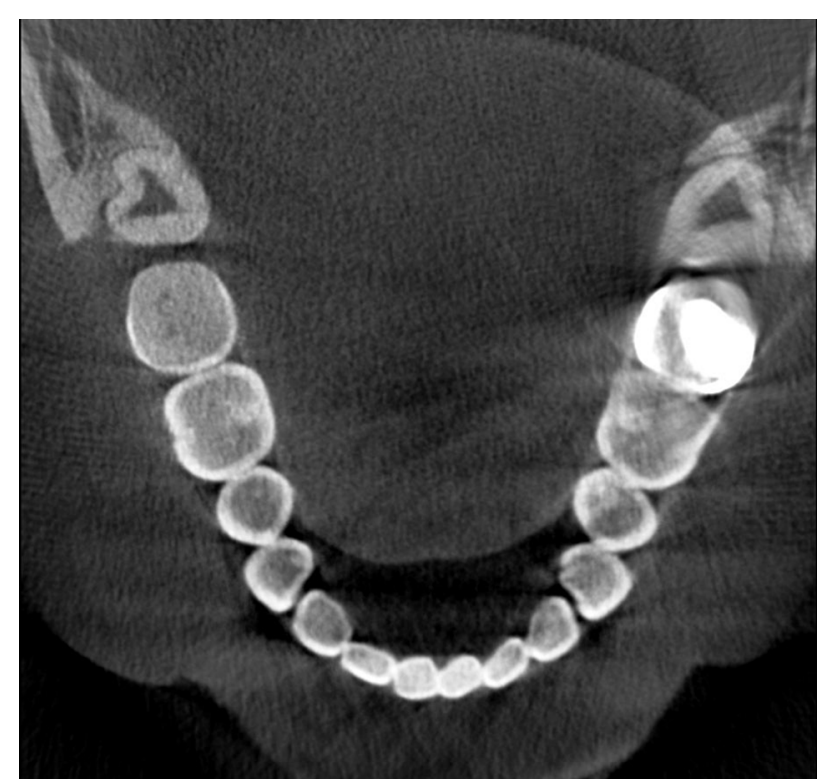

Figure 3. Metal restoration artefact seen on axial image. 


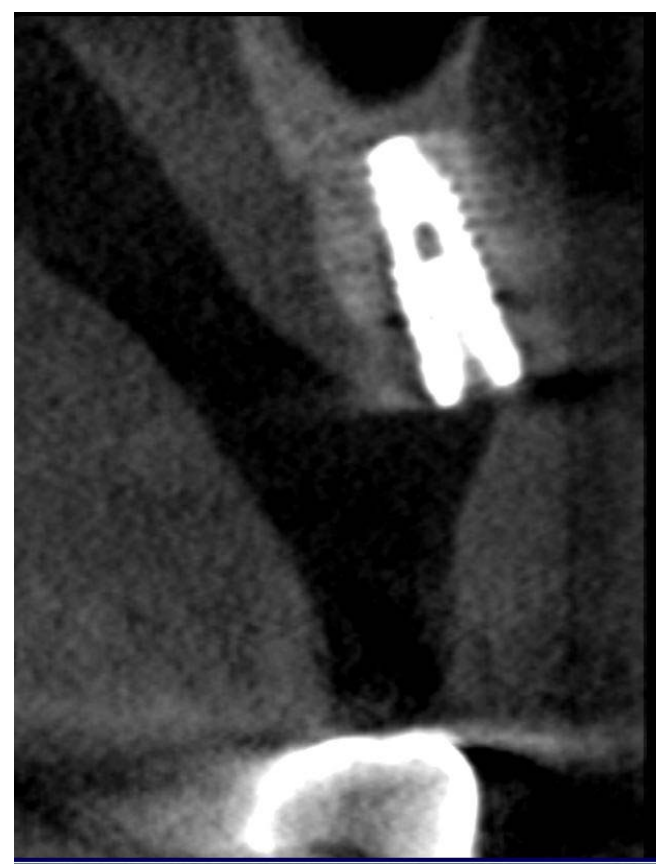

(a)

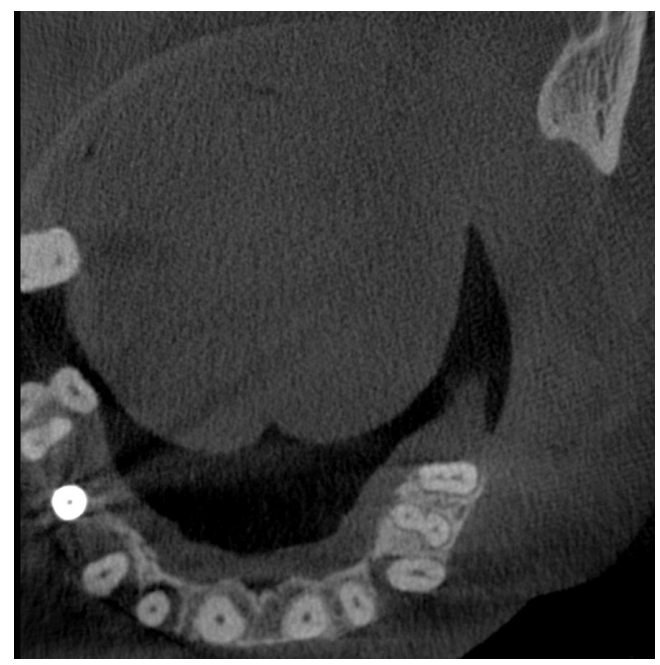

(b)

Figure 4. Implant artefact on axial and cross sectional image.

metal-tissue interface, which is often the main area of diagnostic interest. Beam hardening correction software should also be used when scanning metal objects to minimize the additional artifacts due to beam hardening [9].

\section{MOTION ARTEFACTS}

Patient motion can cause misregistration artifacts within the image. Because of the relatively long acquisition times (compared to conventional radiography) and volumetric image acquisition, motion artefacts are common in CBCT. These artefacts can be attributed to improper patient stabilization. Small motions cause image blurring and larger physical displacements.

They produce artifacts that appear as double images or ghost images. This results in poor overall image quality. Since the resolutions of the present CBCT are very high, ranging from $0.08 \mathrm{~mm}-0.4 \mathrm{~mm}$, even small motions can have a detrimental effect on image quality. The use of positioning aids is sufficient to prevent voluntary movement in most patients.

\section{SCANNER-BASED ARTEFACTS}

\section{Ring Artefacts}

If one of the detectors is out of calibration on a scanner, the detector will give a consistently erroneous reading at each angular position, resulting in a circular artefact. A scanner with solid-state detectors, where all the detectors are separate entities, is in principle more susceptible to ring artefacts. Rings visible in a uniform phantom or in air might not be visible on a clinical image if a wide window is used. Even if they are visible, they would rarely be confused with disease. However, they can impair the diagnostic quality of an image, and this is particularly likely when central detectors are affected, creating a dark smudge at the center of the image. Currently there is no evidence of ring artefacts on CBCT machine in dental radiology literature [9].

\section{CONCLUSION}

Artefacts originate from a range of sources and can degrade the quality of a CBCT images to varying degrees. Design features incorporated into modern machines minimize some types of artifact, and some can be partially corrected by the scanner software like MARS. However, there are many instances where careful patient positioning and the optimum selection of scan parameters are the most important factors in avoiding image artefact.

\section{REFERENCES}

[1] Guerrero, M.E., Jacobs, R., Loubele, M., et al. (2006) State-of-the-art on cone beam CT imaging for preoperative planning of implant placement. Clinical Oral Investigations, 10, 1-7. doi:10.1007/s00784-005-0031-2

[2] Drage, N.A. and Sivarajasingam, V. (2009) The use of cone beam computed tomography inthe management of isolated orbital floor fractures. British Journal of Oral and Maxillofacial Surgery, 47, 65-66. doi:10.1016/j.bjoms.2008.05.005

[3] Holberg, C., Steinhauser, S., Geis, P., et al. (2005) Conebeam computed tomography in orthodontics: Benefits and limitations. Journal of Orofacial Orthopedics, 66, 434-444. doi:10.1007/s00056-005-0519-z

[4] Garrett, B.J., Caruso, J.M., Rungcharassaeng, K., et al. (2008) Skeletal effects to the maxilla after rapid maxil- 
lary expansion assessed with cone-beam computed tomography. American Journal of Orthodontics and Dentofacial Orthopedics, 134, 8-9.

doi:10.1016/j.ajodo.2008.06.004

[5] Misch, K.A., Yi, E.S. and Sarment, D.P. (2006) Accuracy of cone beam computed tomography for periodontal defect measurements. Journal of Periodontology, 77, 12611266. doi:10.1902/jop.2006.050367

[6] Honda, K., Arai, Y., Kashima, M., et al. (2004) Evaluation of the usefulness of the limited cone-beamCT(3DX) in the assessment of the thickness of the roof of the glenoid fossa of the temporomandibular joint. Dentomaxillofacial Radiology, 33, 391-395.

doi: $10.1259 / \mathrm{dmfr} / 54316470$

[7] Bianchi, S., Anglesio, S., Castellano, S., Rizzi, L. and Ragona, R. (2001) Absorbed doses and risk in implant planning: Comparison between spiral CT and cone beam CT. Dentomaxillofacial Radiology, 30, S28.

[8] Tsiklakis, K., Donta, C., Gavala, S., Karayianni, K., Kamenopoulou, V. and Hourdakis, C.J. (2005) Dose reduction in maxillofacial imaging using low dose cone beam CT. European Journal of Radiology, 56, 413-417. doi:10.1016/j.ejrad.2005.05.011

[9] Barrett, J.F. and Keat, N. (2004) Artifacts in CT: Recognition and avoidance. Radiographics, 24, 1679-1691. doi:10.1148/rg.246045065

[10] Hunter, A.K. and McDavid, W.C. (2012) Characterization and correction of cupping effect artefacts in cone beam CT. Dentomaxillofacial Radiology, 41, 217-223. doi:10.1259/dmfr/19015946

[11] Miracle, A.C. and Mukerji, S.K. (2009) Conebeam CT of the head and neck, Part 1: Physical principles. American Journal of Neuroradiology, 30, 1088-1095. doi:10.3174/ajnr.A1653

[12] McDavid, W.D., Waggener, R.G., Payne, W.H. and Dennis, M.J. (1975) Spectral effects on three-dimensional reconstruction from x-rays. Medical Physics, 2, 321-324. doi:10.1118/1.594200

[13] Brooks, R.A. and Di Chiro, G. (1976) Beam hardening in $\mathrm{X}$-ray reconstructive tomography. Physics in Medicine and Biology, 21, 390-398. doi:10.1088/0031-9155/21/3/004

[14] Lehr, J.L. (1983) Truncated-view artifacts: Clinical importance on CT. American Journal of Roentgenology, 141, 183-191. doi:10.2214/ajr.141.1.183

[15] Bryant, J.A., Drage, N.A. and Richmond, S. (2008) Study of the scan uniformity from an i-CAT cone beam CT dental imaging system. Dentomaxillofacial Radiology, 37, 365-374. doi:10.1259/dmfr/13227258

[16] Katsumata, A., Hirukawa, A., Okumura, S., Naitoh, M., Fujishita, M., Ariji, E., et al. (2009) Relationship between density variability and imaging volume size in cone-beam computerized tomographic scanning of the maxillofacial region: An in vitro study. Oral Surgery, Oral Medicine, Oral Pathology, Oral Radiology, and Endodontics, 107, 420-425. doi:10.1016/j.tripleo.2008.05.049

[17] Jennings, R.J. (1988) A method for comparing beamhardening filter materials for diagnostic radiology. Medical Physics, 15, 588-599. doi:10.1118/1.596210

[18] Meganck, J.A., Kozloff, K.M., Thornton, M.M., Broski, S.M. and Goldstein, S.A. (2009) Beam hardening artifacts in micro-CT scanning can be reduced by X-ray beam filtration and the resulting images can be used to accurately measure BMD. Bone, 45, 1104-1116. doi:10.1016/j.bone.2009.07.078

[19] Graham, S.A., Moseley, D.J., Siewerdsen, J.H., et al. (2007) Compensators for dose and scatter management in cone-beam computed tomography. Medical Physics, 34, 2691-2703. doi:10.1118/1.2740466

[20] Gupta, R., Grasruck, M., Suess, C., et al. (2006) Ultrahigh resolution flat-panel volume CT: Fundamental principles, design architecture, and system characterization. European Radiology, 16, 1191-1205. doi:10.1007/s00330-006-0156-y

[21] Ning, R., Chen, B., Yu, R., et al. (2000) Flat panel detector-based cone-beam volume CT angiography imaging: System evaluation. IEEE Transactions on Medical Imaging, 19, 949-963. doi:10.1109/42.887842

[22] Neitzel, U. (1992) Grids or air gaps for scatter reduction in digital radiography: A model calculation. Medical Physics, 19, 475-481. doi:10.1118/1.596836

[23] Siewerdsen, J.H., Moseley, D.J., Bakhtiar, B., et al. (2004) The influence of antiscatter grids on soft-tissue detectability in cone-beam computed tomography with flatpanel detectors. Medical Physics, 31, 3506-3520. doi:10.1118/1.1819789

[24] Nickoloff, E.L., Lu, Z.F., Dutta, A., et al. (2007) Influence of flat-panel fluoroscopic equipment variables on cardiac radiation doses. CardioVascular and Interventional Radiology, 30, 169-176. doi:10.1007/s00270-006-0096-6

[25] Orth, R.C., Wallace, M.J. and Kuo, M.D. (For the Technology Assessment Committee of the Society of Interventional Radiology) (2008) C-arm cone-beam CT: General principles and technical considerations for use in interventional radiology. Journal of Vascular and Interventional Radiology, 19, 814-820. doi:10.1016/j.jvir.2008.02.002

[26] Dorfler, A., Struffert, T., Engelhorn, T., et al. (2008) Rotati onal flat-panel computed tomography in diagnostic and interventional neuroradiology. Rofo, 180, 891-898.

[27] Jarry, G., Graham, S.A., Moseley, D.J., et al. (2006) Characterization of scattered radiation in $\mathrm{kV}$ CBCT images using Monte Carlo simulations. Medical Physics, 33, 43204329. doi:10.1118/1.2358324 